1 Diabetes Research Centre, University of Leicester, Leicester, UK

2 Nuffield Department of Primary Care Health Sciences, University of Oxford Oxford, UK

Correspondence to: K Khunti kk22@le.ac.uk

Cite this as: BMJ 2020;371:m3793 http://dx.doi.org/10.1136/bmj.m3793

Published: 05 October 2020

\section{Pandemic threatens primary care for long term conditions}

\author{
Change is required to ensure effective care during and after covid-19 \\ Louis S Levene, ${ }^{1}$ Samuel Seidu, ${ }^{1}$ Trish Greenhalgh, ${ }^{2}$ Kamlesh Khunti
}

During the height of the covid-19 pandemic, attention was diverted away from primary care's key roles in early detection of disease and management of long term conditions. However, the core primary healthcare functions of accessible first contact, comprehensiveness, coordination, continuity, and person centredness were challenged even before this. ${ }^{1}$ General practices' workloads have steadily risen in the past decade. In England between 2010-11 and 2019, the overall number of consultations per registered patient each year rose from 4.29 to $5.17^{23}$ in a growing population.

Comparing April to August 2020 with the same period in 2019, the total number of general practice appointments in England fell by 20.8\%, from 120.66 million to 95.52 million. ${ }^{4}$ Although these statistics should be treated cautiously, they suggest that substantially fewer planned care appointments took place in 2020. The resulting backlog, combined with the prioritisation of covid-19 in secondary care, has led to further shortfalls in the care of non-covid illness.

Between 27 March and 5 June 2020, most excess mortality was associated with covid-19. ${ }^{5}$ However, delays in diagnosis and suboptimal management of people with long term conditions will cause rises in non-covid mortality, particularly from cancer and cardiac disease. ${ }^{6}$ If lower consulting rates persist and the backlog of planned care is not cleared, people with long term conditions are more likely to experience adverse outcomes, including death.

Patients, healthcare staff, and policy makers are now recognising that the fluctuating health needs of populations will require changes in how primary healthcare is delivered beyond the covid-19 pandemic. Any changes should aim to maximise the capacity and effectiveness of primary care but must also ensure that health inequalities are narrowed not widened, such as the ethnic disparities in infection and mortality seen with covid-19. Several areas should be considered.

\section{Priorities for action}

Firstly, appointments systems may need reviewing. Telephone triage and remote consultations have increased since the start of the pandemic. ${ }^{4}$ Although telehealth reduces patients' direct exposure to infection, it cannot entirely replace face-to-face contacts. Physical examinations are still needed to make certain diagnoses and, more generally, to enhance the human connections between patients and clinicians. Unanticipated findings occasionally save lives. ${ }^{7}$ Digital exclusion of those most in need of healthcare could compound the inverse care law, ${ }^{8}$ since many long term conditions are strongly associated with social determinants of health. ${ }^{9}$ Research should be done to identify solutions for patients who are unable or unwilling to connect with health services remotely. Patients and clinicians also need educating on the effective use of telehealth and remote consultations. Patients should be actively encouraged, where possible, to self-manage self-limiting minor ailments. Professional training should include clear guidance to better prepare healthcare professionals for new ways of working.

Next, further underfunded shifting of patients' care from secondary care to the community must be avoided, allowing overburdened primary care teams to prioritise a worsening backlog of deferred care. In addition, there should be better coordinated and more proactive engagement with staff and carers of patients in care homes or receiving care at home. Use of detailed advanced care plans and agreed pathways for seeking and providing professional help can ensure that patients remain safe, carers feel supported, and demands on practices are balanced.Policy makers and commissioning and regulatory entities should also review their demands on primary care to reduce unnecessary bureaucracy, retaining only essential tasks that are fully justified. For example, the onerous expectation of coroners' offices that general practitioners must have seen a patient within two weeks before death could be modified to accept video reviews within an extended time frame. Recent changes to reduce "red tape" will streamline care, free up professional time, and improve morale.

Evidence suggests that use of point-of-care testing to monitor intermediate markers of chronic disease, such as lipid profiles, glycated haemoglobin, and albumin-creatinine ratios improves practice workflow and increases satisfaction of clinicians, other primary care staff, and patients. ${ }^{10} 11$ Point-of-care testing might also reduce costs, save time, and minimise non-attendance-freeing up valuable clinical time, and research should now be done on these important outcomes.

Finally, recruitment and retention of primary care clinicians must improve. There were 600 (1.8\%) fewer full-time-equivalent general practitioners in June 2020 than in June 2019. ${ }^{12}$ Concerns about workload and remuneration need to be addressed. Optimising the role of nurse practitioners, pharmacists, paramedics, and physician assistants can help in areas such as monitoring chronic disease, care planning, medication reviews and reconciliations, and even home visiting for minor illnesses in patients unable to leave their home.

It is too early to predict what the "new normal" will look like. We must learn the important lessons from 
covid-19 and previous pandemics to avoid suboptimal management of long term conditions contributing to avoidable excess mortality in the medium term. Primary care must be sustainable, with a workload that is manageable, safe, and equitable. Clear, realistic, and fully resourced priorities must now be agreed through collaboration between the public, providers, and commissioners.

Competing interests: We have read and understood BMJ policy on declaration of interests and declare the following interests: KK is member of Independent SAGE and chair of the SAGE subgroup on ethnicity and covid-19. KK, SS, and LSL are supported by the National Institute for Health Research (NIHR) Applied Research Collaboration East Midlands (ARC EM) and the NIHR Leicester Biomedical Research Centre (BRC)

Provenance and peer review: Not commissioned; externally peer reviewed.

1 Dunlop C, Howe A, Li D, Allen LN. The coronavirus outbreak: the central role of primary care in emergency preparedness and response. BJGP Open 2020;4:bjgpopen20X101041. doi: 10.3399/bjgpopen20X101041 pmid: 31992543

2 King's Fund. Is general practice in crisis? Big election questions. 2018. https://www.kingsfund.org.uk/publications/articles/big-election-questions-gp-crisis

3 NHS Digital. Appointments in general practice: comparing 2018 to 2019. 2020. https://digital.nhs.uk/data-and-information/publications/statistical/appointments-in-general-practice/december-2019/page1

$4 \quad$ NHS Digital. Appointments in general practice August 2020-experimental statistics. 2020. https://digital.nhs.uk/data-and-information/publications/statistical/appointments-in-generalpractice/august-2020

5 Office for National Statistics. Deaths registered weekly in England and Wales, provisional: week ending 21 August 2020. 2020. https://www.ons.gov.uk/peoplepopulationandcommunity/birthsdeathsandmarriages/deaths/bulletins/deathsregisteredweeklyinenglandandwalesprovisional/weekending21august2020

6 Tracking covid-19 excess deaths across countries. Economist, 28 Aug 2020. https://www.economist.com/graphic-detail/2020/07/15/tracking-covid-19-excess-deaths-acrosscountries

7 Hyman P. The disappearance of the primary care physical examination-losing touch. JAMA Intern Med 2020. doi: 10.1001/jamainternmed.2020.3546 pmid: 32832987

$8 \quad$ Hart JT. The inverse care law. Lancet 1971;1:405-12. doi: 10.1016/S0140-6736(71)92410-X pmid: 4100731

9 Marmot M, Bell R. Social determinants and non-communicable diseases: time for integrated action. BM/2019;364:1251. doi: 10.1136/bmi.|251 pmid: 30692093

10 Patzer KH, Ardjomand P, Göhring K, etal. Implementation of HbA1c point of care testing in 3 German Medical Practices: impact on workflow and physician, staff, and patient satisfaction. I Diabetes Sci Technol 2018;12:687-94. doi: 10.1177/1932296818759690 pmid: 29532694

11 Kanc K, Komel J, Kos M, Wagner J. H(ome)bA1c testing and telemedicine: High satisfaction of people with diabetes for diabetes management during COVID-19 lockdown. Diabetes Res Clin Pract 2020;166:108285. doi: 10.1016/j.diabres.2020.108285 pmid: 32592835

12 NHS Digital. General practice workforce-30 June 2020. Official statistics. 2020. https://digital.nhs.uk/data-and-information/publications/statistical/general-and-personal-medical-services/30june-2020

This article is made freely available for use in accordance with BMJ's website terms and conditions for the duration of the covid-19 pandemic or until otherwise determined by BMJ. You may use, download and print the article for any lawful, non-commercial purpose (including text and data mining) provided that all copyright notices and trade marks are retained. 